\title{
MULTIPLE COMMUTATOR FORMULAS
}

\author{
R. HAZRAT AND Z. ZHANG
}

\begin{abstract}
Let $A$ be a quasi-finite $R$-algebra (i.e., a direct limit of module finite algebras) with identity. Let $I_{i}, i=0, \ldots, m$, be two-sided ideals of $A, \operatorname{GL}_{n}\left(A, I_{i}\right)$ the principal congruence subgroup of level $I_{i}$ in $\mathrm{GL}_{n}(A)$ and $E_{n}\left(A, I_{i}\right)$ be the relative elementary subgroup of level $I_{i}$. We prove a multiple commutator formula

$$
\begin{aligned}
{\left[E_{n}\left(A, I_{0}\right), \mathrm{GL}_{n}\left(A, I_{1}\right),\right.} & \left.\mathrm{GL}_{n}\left(A, I_{2}\right), \ldots, \mathrm{GL}_{n}\left(A, I_{m}\right)\right] \\
= & {\left[E_{n}\left(A, I_{0}\right), E_{n}\left(A, I_{1}\right), E_{n}\left(A, I_{2}\right), \ldots, E_{n}\left(A, I_{m}\right)\right], }
\end{aligned}
$$

which is a broad generalization of the standard commutator formulas. This result contains all the published results of commutator formulas over commutative rings and answers a problem posed by A. Stepanov and N. Vavilov (cf. Problem 4 in [24]).
\end{abstract}

\section{INTRODUCTION}

Let $A$ be an associative ring with $1, \mathrm{GL}_{n}(A)$ the general linear group of degree $n$ over $A$, and let $E_{n}(A)$ be its elementary subgroup. For a two-sided ideal $I$ of $A$, we denote the principal congruence subgroup of level $I$ by $\operatorname{GL}_{n}(A, I)$ and the relative elementary subgroup of level $I$ by $E_{n}(A, I)$ (see $\S 1.4$ ).

One of the major contributions towards non-stable $K$-theory of rings is the work of Suslin [21, 23] who proved that if $A$ is a module finite ring namely, a ring that is finitely generated as module over its center, and $n \geq 3$ then $E_{n}(A)$ is a normal subgroup of $\mathrm{GL}_{n}(A)$. Thus the non-stable $K_{1}$, i.e., $\mathrm{GL}_{n}(A) / E_{n}(A)$, can be defined. Later Borevich and Vavilov [6] and Vaserstein [26], independently, building on Suslin's method established the standard commutator formula:

Theorem 1 (Suslin, Borevich-Vavilov, Vaserstein). Let $A$ be a module finite ring, I a two-sided ideal of $A$ and $n \geq 3$. Then $E_{n}(A, I)$ is normal in $\mathrm{GL}_{n}(A)$, i.e.,

$$
\left[E_{n}(A, I), \mathrm{GL}_{n}(A)\right]=E_{n}(A, I) \text {. }
$$

Furthermore

$$
\left[E_{n}(A), \mathrm{GL}_{n}(A, I)\right]=E_{n}(A, I) .
$$

One natural question raised here is whether one has a "finer" mixed commutater formulas involving two ideals. In fact this had already been established by Bass for general linear groups of degrees sufficiently larger than the stable rank when he proved his celebrated classification of subgroups of $\mathrm{GL}_{n}$ normalized by $E_{n}$ (see [5, Theorem 4.2]).

Theorem 2 (Bass). Let $A$ be a ring, $I, J$ two-sided ideals of $A$ and $n \geq \max (\operatorname{sr}(R)+1,3)$. Then

$$
\left[E_{n}(A, I), \mathrm{GL}_{n}(A, J)\right]=\left[E_{n}(A, I), E_{n}(A, J)\right] .
$$

Later Mason and Stothers building on Bass' result prove ([18, Theorem 3.6, Corollary 3.9], and [16, Theorem 1.3]):

Theorem 3 (Mason-Stothers). Let $A$ be a ring, $I, J$ two-sided ideals of $A$ and $n \geq \max (\operatorname{sr}(R)+1,3)$. Then

$$
\left[\mathrm{GL}_{n}(A, I), \mathrm{GL}_{n}(A, J)\right]=\left[E_{n}(A, I), E_{n}(A, J)\right] .
$$

The first author acknowledges the support of EPSRC (Grant EP/I007784/1). The second author acknowledges the support of NSFC (Grant 10971011). The authors thank Nikolai Vavilov for suggesting the topic of the paper to them, and Anthony Bak for very useful discussions. 
There are (counter)examples that the Mason-Strothers Theorem does not hold for general module finite rings [1]. However recently Stepanov and Vavilov [24, 25] proved Bass' Theorem 2 for any commutative ring and $n \geq 3$ and the authors using Bak's localization and patching method extend it to all module finite rings [13]. We refer to this as the generalized commutator formula. In [24] it is asked whether one can establish a multiple commutator formula, namely for a commutative ring $R, I_{i}, i=0, \ldots, m$, ideals of $R$ and $n \geq 3$, whether

$$
\begin{aligned}
{\left[E_{n}\left(R, I_{0}\right), \mathrm{GL}_{n}\left(R, I_{1}\right),\right.} & \left.\mathrm{GL}_{n}\left(R, I_{2}\right), \ldots, \mathrm{GL}_{n}\left(R, I_{m}\right)\right] \\
& =\left[E_{n}\left(R, I_{0}\right), E_{n}\left(R, I_{1}\right), E_{n}\left(R, I_{2}\right), \ldots, E_{n}\left(R, I_{m}\right)\right],
\end{aligned}
$$

is valid which is a broad generalization of the standard/generalized commutator formulas. Here for simplicity we write $\left[A_{1}, A_{2}, A_{3}, \ldots, A_{n}\right]$ for $\left[\ldots\left[\left[A_{1}, A_{2}\right], A_{3}\right], \ldots, A_{n}\right]$ (see $\S 1.3$ ). Questions of this type arise from the study of subnormal subgroups of $\mathrm{GL}_{n}$ from one hand and the nilpotent structure of nonstable $K_{1}$ from the other hand (see [12, $\$ 10$ and $\S 12]$ for a survey on these topics).

In this paper we prove Formula (1) for quasi finite rings (which include module finite and commutative rings) (see Corollary 15). In particular this result contains all the published results of commutator formulas over commutative rings. In fact in Theorem 17 we show that the multiple commutator formulas are valid for any meaningful way of the distribution of commutators.

To establish these results, we use the general "yoga of commutators" which are developed in [13] and [14] based on the work of Bak on the localization and patching in general linear groups (see [1, 15] and [12, §13]). In order to utilize this method, one needs to overcome two problems. First to devise an appropriate conjugation calculus to approach the identity (1) and then perform the actual calculations. Both of these are equally challenging as the nature of conjugation calculus depends on the problem in hand. In fact the term yoga of commutators is chosen to stress the overwhelming feeling of technical strain and exertion. However once this is done for general linear groups, one can adapt the approach to more complex settings, such as general quadratic groups and Chevalley groups. These shall be established in a sequel to this paper.

\section{Preliminaries}

In this section we fix some notations. At the same time, we list some preliminary results concerning the localization and patching method without proofs. We refer to Bak's original paper [1] or a survey version in $[12, \S 13]$ for details.

1.1. Let $R$ be a commutative ring with $1, S$ a multiplicative closed system in $R$ and $A$ an $R$-algebra. Then $S^{-1} R$ and $S^{-1} A$ denote the corresponding localization. In the current paper, we mostly use localization with respect to the following two types of multiplicative systems.

1.) For any $s \in R$, the multiplicative system generated by $s$ is defined as

$$
\langle s\rangle=\left\{1, s, s^{2}, \ldots\right\} .
$$

The localization with respect to multiplicative system $\langle s\rangle$ is usually denoted by $R_{s}$ and $A_{s}$. Note that, for any $\alpha \in R_{s}$, there exists an integer $n$ and an element $a \in R$ such that $\alpha=a / s^{n}$.

2.) If $\mathfrak{m}$ is a maximal ideal of $R$, and $S=R \backslash \mathfrak{m}$ a multiplicative system, then we denote the localization with respect to $S$ by $R_{\mathfrak{m}}$ and $A_{\mathfrak{m}}$.

For a multiplicative system $S$, the canonical localization map with respect to $S$ is denoted by $\theta_{S}: R \rightarrow$ $S^{-1} R$. For the special cases mentioned above, we write $\theta_{s}: R \rightarrow R_{s}$ and $\theta_{M}: R \rightarrow R_{M}$, respectively.

1.2. An $R$-algebra $A$ is called module finite over $R$, if $A$ is finitely generated as an $R$-module. An $R$ algebra $A$ is called quasi-finite over $R$ if there is a direct system of module finite $R$-subalgebras $A_{i}$ of $A$ such that $\lim _{\longrightarrow} A_{i}=A$.

Proposition 4. An R-algebra $A$ is quasi-finite over $R$ if and only if it satisfies the following equivalent conditions: 
(1) There is a direct system of subalgebras $A_{i} / R_{i}$ of $A$ such that each $A_{i}$ is module finite over $R_{i}$ and such that $\lim _{i} R_{i}=R$ and $\lim _{i} A_{i}=A$.

(2) There is a direct system of subalgebras $A_{i} / R_{i}$ of $A$ such that each $A_{i}$ is module finite over $R_{i}$ and each $R_{i}$ is finitely generated as a $\mathbb{Z}$-algebra and such that $\lim _{\longrightarrow} R_{i}=R$ and $\lim _{\longrightarrow} A_{i}=A$.

1.3. Let $G$ be a group. For any $x, y \in G,{ }^{x} y=x y x^{-1}$ denotes the left $x$-conjugate of $y$. Let $[x, y]=$ $x y x^{-1} y^{-1}$ denote the commutator of $x$ and $y$. Sometimes the double commutator $[[x, y], z]$ will be denoted simply by $[x, y, z]$ and

$$
[[A, B], C]=[A, B, C] .
$$

Thus we write $\left[A_{1}, A_{2}, A_{3}, \ldots, A_{n}\right]$ for $\left[\ldots\left[\left[A_{1}, A_{2}\right], A_{3}\right], \ldots, A_{n}\right]$ and call it the standard form of the multiple commutator formulas.

The following formulas will be used frequently (sometimes without giving a reference to them),

(C1) $[x, y z]=[x, y]\left({ }^{y}[x, z]\right)$;

$\left(\mathrm{C} 1^{+}\right)$An easy induction, using identity $(\mathrm{C} 1)$, shows that

$$
\left[x, \prod_{i=1}^{k} u_{i}\right]=\prod_{i=1}^{k} \prod_{j=1}^{i-1} u_{j}\left[x, u_{i}\right]
$$

where by convention $\prod_{j=1}^{0} u_{j}=1$.

(C2) $[x y, z]=\left({ }^{x}[y, z]\right)[x, z]$;

$\left(\mathrm{C} 2^{+}\right)$As in $\left(\mathrm{C} 1^{+}\right)$, we have

$$
\left[\prod_{i=1}^{k} u_{i}, x\right]=\prod_{i=1}^{k} \prod_{j=1}^{k-i} u_{j}\left[u_{k-i+1}, x\right]
$$

(C3) (the Hall-Witt identity): ${ }^{x}\left[\left[x^{-1}, y\right], z\right] z\left[\left[z^{-1}, x\right], y\right]{ }^{y}\left[\left[y^{-1}, z\right], x\right]=1$;

(C4) $\left[x,{ }^{y} z\right]={ }^{y}\left[y^{-1} x, z\right]$;

(C5) $\left[{ }^{y} x, z\right]={ }^{y}\left[x, y^{-1} z\right]$.

(C6) If $H$ and $K$ are subgroups of $G$, then $[H, K]=[K, H]$.

1.4. For any associative ring $A, \mathrm{GL}_{n}(A)$ denotes the general linear group of $A$, and $E_{n}(A)$ denotes the elementary subgroup of $\mathrm{GL}_{n}(A)$. Let $I$ be any two-sided ideal of $A$. If $\rho_{I}$ denotes the natural ring homomorphism $A \rightarrow A / I$, then $\rho_{I}$ induces a group homomorphism, denoted also by $\rho_{I}, \rho_{I}: \mathrm{GL}_{n}(A) \rightarrow$ $\mathrm{GL}_{n}(A / I)$. The congruence subgroup of level $I$ is defined as $\mathrm{GL}_{n}(A, I)=\operatorname{ker}\left(\rho_{I}: \mathrm{GL}_{n}(A) \rightarrow \mathrm{GL}_{n}(A / I)\right)$. The elementary subgroup of level $I$ is, by definition, the subgroup generated by all elementary matrices $e_{i, j}(\alpha)$ with $\alpha \in I$. The normal closure of $E_{n}(I)$ in $E_{n}(A)$, the relative elementary subgroup of level $I$, is denoted by $E_{n}(A, I)$. We use $E_{n}^{L}(I)$ to denote the subset of $E_{n}(I)$, which can be represented as the product $L$ elementary matrices. $E_{n}^{L}(I)$ is not necessarily a group.

We have the following relations among elementary matrices which will be used in the paper:

(E1) $e_{i, j}(a) e_{i, j}(b)=e_{i, j}(a+b)$.

(E2) $\left[e_{i, j}(a), e_{k, l}(b)\right]=1$ if $i \neq l, j \neq k$.

(E3) $\left[e_{i, j}(a), e_{j, k}(b)\right]=e_{i, k}(a b)$ if $i \neq k$.

1.5. $\mathrm{GL}_{n}$ and $E_{n}$ define two functors from the category of associative rings to the category of groups. These functors commute with direct limits. In another words, let $A_{i}$ be an inductive system of rings, and $A=\lim _{i} A_{i}$. Then

$$
\mathrm{GL}_{n}(A)=\mathrm{GL}_{n}\left(\stackrel{\lim }{\longrightarrow} A_{i}\right) \cong \lim _{\longrightarrow} \mathrm{GL}_{n}\left(A_{i}\right) \text { and } \quad E_{n}\left(\underline{\lim } A_{i}\right) \cong \underline{\lim } E_{n}\left(A_{i}\right) .
$$

Also, if $J$ is an ideal of $A$, then there are ideals $J_{i}$ of $A_{i}$ such that $J=\lim _{\longrightarrow} J_{i}$ and

$$
\mathrm{GL}_{n}(A, J)=\mathrm{GL}_{n}\left(\lim _{\longrightarrow} A_{i}, \lim _{\longrightarrow} J_{i}\right) \cong \lim _{\longrightarrow} \mathrm{GL}_{n}\left(A_{i}, J_{i}\right) .
$$


By Proposition 4 and the above observation, we may reduce some of our problems to the case of the Noetherian rings. Let $S$ be a multiplicative system in $R, R_{s}$ with $s \in S$ is an inductive system with respect to the localization map : $\theta_{t}: R_{s} \rightarrow R_{s t}$. If $\mathcal{F}$ is a functor commuting with direct limits (here $\mathrm{GL}_{n}$ and $E_{n}$ ), then

$$
\mathcal{F}\left(S^{-1} R\right)=\lim _{\longrightarrow} F\left(R_{s}\right) .
$$

This allows us to reduce our problems in any localization to the localization in one element. Starting from Section 2, we will be working in the $\operatorname{ring} A_{t}$. However, eventually we need to return to the $\operatorname{ring} A$. The following Lemma provides a way to "pull back" elements from $\mathrm{GL}_{n}\left(A_{t}\right)$ to $\mathrm{GL}_{n}(A)$.

Lemma 5. [1, Lemma 4.10] Let $A$ be a module finite $R$-algebra, where $R$ is a commutative Noetherian ring. Then for any $t \in R$, there exists a positive integer $l$ such that the homomorphism $\theta_{t}: \mathrm{GL}_{n}\left(A, t^{l} A\right) \longrightarrow$ $\mathrm{GL}_{n}\left(A_{t}\right)$ is injective.

Definition 6. Let $A$ be an $R$-algebra, $I$ a two-sided ideal of $A, t \in R$, and $l$ a positive integer. Define $E_{n}\left(t^{l} A, t^{l} I\right)$ to be a subgroup of $E_{n}\left(A, t^{l} I\right)$ generated by

$$
{ }^{e} e_{i, j}\left(t^{l} \alpha\right) \text { for all } \alpha \in I, e \in E_{n}\left(t^{l} A\right) \text { and } 1 \leq i \neq j \leq n .
$$

Here by $t^{l} I$, we are considering the image of $t \in R$ in $A$ under the algebra structure homomorphism. It is clear that $t^{l} I$ is also an ideal of $A$.

For any element $\alpha \in A$, we use $E_{n}\left(t^{l} A, t^{l} \alpha\right)$ to denote the subgroup generated by

$$
{ }^{e} e_{i, j}\left(t^{l} \alpha\right) \text { for all } e \in E_{n}\left(t^{l} A\right) \text { and } 1 \leq i, j \leq n .
$$

From the definition, it is clear that $E_{n}\left(t^{l} A, t^{l} I\right)$ is normalized by $E_{n}\left(t^{l} A\right)$. This will be used throughout out the calculations. Also, by Lemma 5 , both $E_{n}\left(t^{l} A, t^{l} I\right)$ and $E_{n}\left(t^{l} A, t^{l} \alpha\right)$ are embedded in $\mathrm{GL}_{n}\left(A_{t}\right)$ for a sufficiently large integer $l$. This fact will be used in Theorem 14 .

1.6. Finally we need the following elementary conjugation calculus, Lemmas 7, 8 and 11 from [13], respectively. Note that in Equations 2, 3 and 4 the calculations take place in the group $E_{n}\left(A_{t}\right)$.

Lemma 7 (cf. [13]). Let $A$ be a module finite $R$-algebra, $I, J$ two-sided ideals of $A, a, b, c \in A$ and $t \in R$. If $m, l$ are given, there is an integer $p$ such that

$$
E_{n}^{1}\left(\frac{c}{t^{m}}\right) E_{n}\left(t^{p} A, t^{p}\langle a\rangle\right) \subseteq E_{n}\left(t^{l} A, t^{l}\langle a\rangle\right),
$$

there is an integer $p$ such that

$$
E_{n}^{1}\left(\frac{c}{t^{m}}\right)\left[E_{n}\left(t^{p} A, t^{p}\langle a\rangle\right), E_{n}\left(t^{p} A, t^{p}\langle b\rangle\right)\right] \subseteq\left[E_{n}\left(t^{l} A, t^{l}\langle a\rangle\right), E_{n}\left(t^{l} A, t^{l}\langle b\rangle\right)\right],
$$

and there is an integer $p$ such that

$$
\left[E_{n}\left(t^{p} A, t^{p} I\right), E_{n}^{1}\left(\frac{J}{t^{m}}\right)\right] \subseteq\left[E_{n}\left(t^{l} A, t^{l} I\right), E_{n}\left(t^{l} A, t^{l} J\right)\right] .
$$

By Lemma 7, one obtains the following result easily. The proof is left to the reader.

Lemma 8. Let $A$ be a module finite R-algebra, $I, J$ two-sided ideals of $A, a, b, c \in A$ and $t \in R$. If $m, l, L$ are given, there is an integer $p$ such that

$$
\left[E_{n}\left(t^{p} A, t^{p} I\right),{ }^{E_{n}^{L}}\left(\frac{A}{t^{m}}\right) E_{n}^{1}\left(\frac{J}{t^{m}}\right)\right] \subseteq\left[E_{n}\left(t^{l} A, t^{l} I\right), E_{n}\left(t^{l} A, t^{l} J\right)\right]
$$

\section{Commutator subgroups}

In this section we study the relations between multiple commutator subgroups over a quasi-finite algebra. The proofs are heavily depend on the computation in [13] (see Lemma 7). Throughout the section ideals are two sided and we assume $n \geq 3$ for any general linear group $\mathrm{GL}_{n}$.

We record the following well-known lemma originally established by Suslin and Vaserstein (cf. [1, Lemma 4.8]) which is needed in computations. 
Lemma 9. Let $A$ be a ring and I a two-ideal of $A$. Then $E_{n}(A, I)$ is generated as a group by the elements

$$
e_{i, j}(a) e_{j, i}(\alpha) \text {, }
$$

where $i \neq j, a \in A$ and $\alpha \in I$.

Using Lemma 9 it is not hard to prove that $E_{n}\left(A, I^{2}\right) \subseteq E_{n}(I)$ (see [1, Corollary 4.9] and [22, Proposition 2]). This containment can be slightly generalized to the case of two ideals. The following Lemma will be used throughout our calculations.

Lemma 10. Let $A$ be a ring and $I, J$ be two-ideals of $A$. Then

$$
E_{n}(A, I J+J I) \subseteq\left[E_{n}(I), E_{n}(J)\right] \subseteq\left[E_{n}(A, I), E_{n}(A, J)\right] \subseteq \mathrm{GL}_{n}(A, I J+J I) .
$$

Proof. The proof is routine by using Lemma 9 and is left to the reader.

Lemma 11. Let $A$ be a ring and $I, J$ be two-ideals of $A$. Then $\left[E_{n}(A, I), E_{n}(A, J)\right]$ is generated as a group by the elements of the form

$$
{ }^{c}\left[e_{j, i}(\alpha),{ }^{e}{ }_{i, j}(a) e_{j, i}(\beta)\right], \quad{ }^{c}\left[e_{j, i}(\alpha), e_{i, j}(\beta)\right], \quad{ }^{c} e_{i, j}(\alpha \beta), \quad \text { and } \quad{ }^{c} e_{i, j}(\beta \alpha),
$$

where $1 \leq i \neq j \leq n, \alpha \in I, \beta \in J, a \in A$ and $c \in E_{n}(A)$.

Proof. A typical generator of $\left[E_{n}(A, I), E_{n}(A, J)\right]$ is of the form $[e, f]$, where $e \in E_{n}(A, I)$ and $f \in$ $E_{n}(A, J)$. Thanks to Lemma 9 , we may assume that $e$ and $f$ are products of elements the form

$$
e_{i}={ }^{e^{\prime}, q^{\prime}}(a) e_{q^{\prime}, p^{\prime}}(\alpha) \text { and } f_{j}={ }^{e_{p, q}(b)} e_{q, p}(\beta),
$$

where $a, b \in A, \alpha \in I$ and $\beta \in J$, respectively. Applying $\left(\mathrm{C}^{+}\right)$and then $\left(\mathrm{C} 2^{+}\right)$, one gets that $\left[E_{n}(A, I), E_{n}(A, J)\right]$ is generated by the elements of the form

$$
{ }^{c}\left[{ }^{e^{\prime}, j^{\prime}}(a) e_{j^{\prime}, i^{\prime}}(\alpha),{ }^{e_{i, j}(b)} e_{j, i}(\beta)\right],
$$

where $c \in E_{n}(A)$. Furthermore,

$$
{ }^{c}\left[{ }^{e_{i^{\prime}, j^{\prime}}(a)} e_{j^{\prime}, i^{\prime}}(\alpha),{ }^{e_{i, j}(b)} e_{j, i}(\beta)\right]={ }^{c e_{i^{\prime}, j^{\prime}}(a)}\left[e_{j^{\prime}, i^{\prime}}(\alpha),{ }_{i^{\prime}, j^{\prime}}(-a) e_{i, j}(b) e_{j, i}(\beta)\right] .
$$

The normality of $E_{n}(A, J)$ implies that ${ }^{e_{i^{\prime}, j^{\prime}}(-a) e_{i, j}(b)} e_{j, i}(\beta) \in E_{n}(A, J)$, which is a product of ${ }^{e_{p, q}(a)} e_{q, p}(\beta)$, $a \in A$ and $\beta \in J$ by Lemma 9. Again by $\left(\mathrm{C}^{+}\right)$, one reduces the proof to the case of showing that

$$
\left[e_{i^{\prime}, j^{\prime}}(\alpha),{ }^{e_{i, j}(a)} e_{j, i}(\beta)\right]
$$

is a product of the generators listed in (6). We need to consider following cases:

- If $i^{\prime}=j, j^{\prime}=i$ : Then there is nothing to proof.

- if $i^{\prime}=j, j^{\prime} \neq i$ :

$$
\begin{aligned}
{\left[e_{j, j^{\prime}}(\alpha),{ }^{e_{i, j}(a)} e_{j, i}(\beta)\right] } & =e_{i, j}(a)\left[e_{i, j}(-a) e_{j, j^{\prime}}(\alpha), e_{j, i}(\beta)\right] \\
& =e_{i, j}(a)\left[\left[e_{i, j}(-a), e_{j, j^{\prime}}(\alpha)\right] e_{j, j^{\prime}}(\alpha), e_{j, i}(\beta)\right] \\
& =e_{i, j}(a)\left[e_{i, j^{\prime}}(-a \alpha) e_{j, j^{\prime}}(\alpha), e_{j, i}(\beta)\right] .
\end{aligned}
$$

Applying now (C2),

$$
\begin{aligned}
{\left[e_{i, j^{\prime}}(-a \alpha) e_{j, j^{\prime}}(\alpha), e_{j, i}(\beta)\right] } & =\left(e_{i, j^{\prime}}(-a \alpha)\left[e_{j, j^{\prime}}(\alpha), e_{j, i}(\beta)\right]\right)\left[e_{i, j^{\prime}}(-a \alpha), e_{j, i}(\beta)\right] \\
& =\left[e_{i, j^{\prime}}(-a \alpha), e_{j, i}(\beta)\right] \\
& =\left[e_{j, i}(\beta), e_{i, j^{\prime}}(-a \alpha)\right]^{-1} \\
& =e_{j, j^{\prime}}(-\beta a \alpha)^{-1} \\
& =e_{j, j^{\prime}}(\beta a \alpha)
\end{aligned}
$$

Thus

$$
\left[e_{j, j^{\prime}}(\alpha),{ }^{e_{i, j}(a)} e_{j, i}(\beta)\right]={ }^{e_{i, j}(a)} e_{j, j^{\prime}}(\beta a \alpha)
$$

which satisfies the lemma. 
- if $i^{\prime} \neq j, j^{\prime}=i$ : The argument is similar to the previous case.

- if $i^{\prime} \neq j, j^{\prime} \neq i$ : We consider four cases:

- if $i^{\prime}=i, j^{\prime}=j$ :

$$
\left[e_{i, j}(\alpha),{ }^{e_{i, j}(a)} e_{j, i}(\beta)\right]={ }^{e_{i, j}(a)}\left[e_{i, j}(\alpha), e_{j, i}(\beta)\right] .
$$

- if $i^{\prime}=i, j^{\prime} \neq j$ :

$$
\begin{aligned}
{\left[e_{i, j^{\prime}}(\alpha),{ }^{e_{i, j}(a)} e_{j, i}(\beta)\right] } & ={ }^{e_{i, j}(a)}\left[e_{i, j^{\prime}}(\alpha), e_{j, i}(\beta)\right] \\
& ={ }^{e_{i, j}(a)} e_{j, j^{\prime}}(-\beta \alpha) .
\end{aligned}
$$

- if $i^{\prime} \neq i, j^{\prime}=j$ :

$$
\begin{aligned}
{\left[e_{i^{\prime}, j}(\alpha),{ }^{e_{i, j}(a)} e_{j, i}(\beta)\right] } & ={ }^{e_{i, j}(a)}\left[e_{i^{\prime}, j}(\alpha), e_{j, i}(\beta)\right] \\
& ={ }^{e_{i, j}(a)} e_{i, i^{\prime}}(\alpha \beta) .
\end{aligned}
$$

- if $i^{\prime} \neq i, j^{\prime} \neq j$ :

$$
\left[e_{i^{\prime}, j^{\prime}}(\alpha),{ }^{e_{i, j}(a)} e_{j, i}(\beta)\right]=1 \text {. }
$$

This finishes the proof.

Denote by $E_{n}^{L}\left(\frac{A}{t^{m}}, \frac{K}{t^{m}}\right)$ the product of $L$ elements (or fewer) of the form $E_{n}^{1}\left(\frac{A}{t^{m}}\right) E_{n}^{1}\left(\frac{K}{t^{m}}\right)$ (see also $\S 1.4$ ). In the following two Lemmas, as in Lemma 7, all the calculations take place in the fraction ring $A_{t}$ (see §1.6). All the subgroups used in the Lemmas, such as $E_{n}(A, I)$ or $\operatorname{GL}_{n}(A, J)$ are in fact the images of these groups in $\mathrm{GL}_{n}\left(A_{t}\right)$ under the ring homomorphisms $A \rightarrow A_{t}$. This allows us to use Lemmas such as Lemma 10 and the generalized commutator formula on these subgroups, precisely because these are homomorphic images of the similar subgroups in $\mathrm{GL}_{n}(A)$ which Lemma 10, etc. hold.

Lemma 12. Let $A$ be a module finite $R$-algebra, $I, J$ two-sided ideals of $A$, and $t \in R$. If $e \in \mathrm{GL}_{n}\left(A_{t}, J_{t}\right)$, there is an integer $p$ such that for any $g \in \mathrm{GL}_{n}\left(A, t^{p} I\right)$

$$
[e, g] \in \mathrm{GL}_{n}\left(A, t^{l}(I J+J I)\right) .
$$

Proof. Note that all the entries of $g-1$ and $g^{-1}-1$ are in $t^{p} I$ (to emphasize our convention, they are in the image of $t^{p} I$ under the homomorphism $\theta: A \rightarrow A_{t}$ ) and all the entries of $e-1$ and $e^{-1}-1$ are in $J_{t}$. Choose $k \in \mathbb{N}$ such that one can write all the entries of $e-1$ and $e^{-1}-1$ of the form $j / t^{k}, j \in J$. Let

$$
\begin{array}{llll}
g=1+\varepsilon & \text { and } & g^{-1}=1+\varepsilon^{\prime} \\
e=1+\delta & \text { and } & e^{-1}=1+\delta^{\prime} .
\end{array}
$$

A straightforward computation shows that

$$
\begin{aligned}
& \varepsilon+\varepsilon^{\prime}+\varepsilon \varepsilon^{\prime}=\varepsilon+\varepsilon^{\prime}+\varepsilon^{\prime} \varepsilon=0 \\
& \delta+\delta^{\prime}+\delta \delta^{\prime}=\delta+\delta^{\prime}+\delta^{\prime} \delta=0 .
\end{aligned}
$$

By the equalities above, one has

$$
[e, g]=[1+\delta, 1+\varepsilon]=1+\delta^{\prime} \varepsilon^{\prime}+\varepsilon \delta^{\prime}+\varepsilon \delta^{\prime} \varepsilon^{\prime}+\delta \delta^{\prime} \varepsilon^{\prime}+\delta \varepsilon \delta^{\prime}+\delta \varepsilon \delta^{\prime} \varepsilon^{\prime} .
$$

So the entries of $[e, g]-1$ belong to $t^{p-2 k}(I J+J I)$. We finish the proof by choosing $p \geq l+2 k$.

The following lemma is crucial for proving the main result, i.e., Theorem 14 of this paper.

Lemma 13. Let $A$ be a module finite $R$-algebra, $I, J, K$ two-sided ideals of $A$ and $t \in R$. For any given $e_{2} \in E_{n}\left(A_{t}, K_{t}\right)$ and an integer $l$, there is a sufficiently large integer $p$, such that

$$
\left[e_{1}, e_{2}\right] \in\left[\left[E_{n}\left(A, t^{l} I\right), E_{n}\left(A, t^{l} J\right)\right], E_{n}\left(A, t^{l} K\right)\right] .
$$

where $e_{1} \in\left[E_{n}\left(t^{p} I\right), E_{n}(A, J)\right]$. 
Proof. For any given $e_{2} \in E_{n}\left(A_{t}, K_{t}\right)$, one may find some positive integers $m$ and $L$, such that

$$
e_{2} \in E_{n}^{L}\left(\frac{A}{t^{m}}, \frac{K}{t^{m}}\right) .
$$

Applying the identity $\left(\mathrm{C}^{+}\right)$and repeated application of $(2)$ in Lemma 7, we reduce the problem to show that

$$
\left[\left[E_{n}\left(t^{p} I\right), E_{n}(A, J)\right],{ }^{c} e_{i^{\prime}, j^{\prime}}\left(\frac{\gamma}{t^{m}}\right)\right] \subseteq\left[\left[E_{n}\left(A, t^{l} I\right), E_{n}\left(A, t^{l} J\right)\right], E_{n}\left(A, t^{l} K\right)\right],
$$

where $c \in E_{n}^{1}\left(\frac{A}{t^{m}}\right)$ and $\gamma \in K$. We further decompose $e_{i^{\prime}, j^{\prime}}\left(\frac{\gamma}{t^{m}}\right)=\left[e_{i^{\prime}, k}\left(t^{p^{\prime}}\right), e_{k, j^{\prime}}\left(\frac{\gamma}{t^{m+p^{\prime}}}\right)\right]$ for some integer $p^{\prime}$. Then

$$
\left[e_{1},{ }^{c} e_{i^{\prime}, j^{\prime}}\left(\frac{\gamma}{t^{m}}\right)\right]=\left[e_{1},\left[{ }^{c} e_{i^{\prime}, k}\left(t^{p^{\prime}}\right),{ }^{c} e_{k, j^{\prime}}\left(\frac{\gamma}{t^{m+p^{\prime}}}\right)\right]\right] .
$$

We use a variant of the Hall-Witt identity (see (C3))

$$
\left[x,\left[y^{-1}, z\right]\right]=y^{-1} x\left[\left[x^{-1}, y\right], z\right] y^{y^{-1} z}\left[\left[z^{-1}, x\right], y\right],
$$

to obtain

$$
\begin{aligned}
& {\left[e_{1},\left[{ }^{c} e_{i^{\prime}, k}\left(t^{p^{\prime}}\right),{ }^{c} e_{k, j^{\prime}}\left(\frac{\gamma}{t^{m+p^{\prime}}}\right)\right]\right]=} \\
& =y^{-1} x\left[\left[e_{1}^{-1},{ }^{c} e_{i^{\prime}, k}\left(-t^{p^{\prime}}\right)\right],{ }^{c} e_{k, j^{\prime}}\left(\frac{\gamma}{t^{m+p^{\prime}}}\right)\right] \times \\
& y^{-1} z\left[\left[{ }^{c} e_{k, j^{\prime}}\left(\frac{-\gamma}{t^{m+p^{\prime}}}\right), e_{1}\right],{ }^{c} e_{i^{\prime}, k}\left(-t^{p^{\prime}}\right)\right],
\end{aligned}
$$

where $x=e_{1}, y={ }^{c} e_{i^{\prime}, k}\left(-t^{p^{\prime}}\right), z={ }^{c} e_{k, j^{\prime}}\left(\frac{\gamma}{t^{m+p^{\prime}}}\right)$ and as before $c \in E_{n}^{1}\left(\frac{A}{t^{m}}\right) \subseteq E_{n}^{1}\left(\frac{A}{t^{m+p^{\prime}}}\right)$. We will look at each of the two factors of (8) separately.

By (2) in Lemma 7, for any given $p^{\prime \prime}$, one may find a sufficiently large $p^{\prime}$ such that

$$
y={ }^{c} e_{i^{\prime}, k}\left(-t^{p^{\prime}}\right) \in E_{n}\left(t^{p^{\prime \prime}} A, t^{p^{\prime \prime}} A\right) \subseteq E_{n}(A) .
$$

Then

$$
\begin{aligned}
{\left[e_{1}^{-1},{ }^{c} e_{i^{\prime}, k}\left(-t^{p^{\prime}}\right)\right] } & \in\left[\left[E_{n}\left(t^{p} I\right), E_{n}(A, J)\right], E_{n}(A)\right] \\
& \subseteq\left[\operatorname{GL}_{n}\left(A, t^{p}(I J+J I)\right), E_{n}(A)\right] \\
& \subseteq E_{n}\left(A, t^{p}(I J+J I)\right) .
\end{aligned}
$$

Set $p_{1}=p$. Thanks to Lemma 10 ,

$$
E_{n}\left(A, t^{p_{1}}(I J+J I)\right) \subseteq\left[E_{n}\left(t^{\left\lfloor\frac{p_{1}}{2}\right\rfloor} A\right), E_{n}\left(t^{\left\lfloor\frac{p_{1}}{2}\right\rfloor}(I J+J I)\right)\right] \subseteq E_{n}\left(t^{\left\lfloor\frac{p_{1}}{2}\right\rfloor} A, t^{\left\lfloor\frac{p_{1}}{2}\right\rfloor}(I J+J I)\right) .
$$

Hence we obtain that

$$
y^{-1} x\left[\left[e_{1}^{-1},{ }^{c} e_{i^{\prime}, k}\left(-t^{p^{\prime}}\right)\right],{ }^{c} e_{k, j^{\prime}}\left(\frac{\gamma}{t^{m+p^{\prime}}}\right)\right] \in y^{-1} x\left[E_{n}\left(t^{t^{\left.\frac{p_{1}}{2}\right\rfloor}} A, t^{\left.t^{\frac{p_{1}}{2}}\right\rfloor}(I J+J I)\right),{ }^{c} e_{k, j^{\prime}}\left(\frac{\gamma}{t^{m+p^{\prime}}}\right)\right],
$$

where $x \in\left[E_{n}\left(t^{p_{1}} I\right), E_{n}(A, J)\right], y \in E_{n}\left(t^{p^{\prime \prime}} A, t^{p^{\prime \prime}} A\right)$. By Lemma 8, for any given integer $l^{\prime}$ we may find a sufficiently large $p_{1}$, such that

$$
\begin{aligned}
y^{-1} x\left[E_{n}\left(t^{\left\lfloor\frac{p_{1}}{2}\right\rfloor} A, t^{\left.\frac{p_{1}}{2}\right\rfloor}(I J+J I)\right),\right. & \left.{ }^{c} e_{k, j^{\prime}}\left(\frac{\gamma}{t^{m+p^{\prime}}}\right)\right] \in y^{-1} x\left[E_{n}\left(t^{2 l^{\prime}} A, t^{2 l^{\prime}}(I J+J I)\right), E_{n}\left(t^{2 l^{\prime}} A, t^{2 l^{\prime}} K\right)\right] \\
& \subseteq y^{-1} x\left[\left[E_{n}\left(t^{l^{\prime}} A, t^{l^{\prime}} I\right), E_{n}\left(t^{l^{\prime}} A, t^{l^{\prime}} J\right)\right], E_{n}\left(t^{2 l^{\prime}} A, t^{2 l^{\prime}} K\right)\right] \\
& \subseteq y^{-1} x\left[\left[E_{n}\left(t^{l^{\prime}} A, t^{l^{\prime}} I\right), E_{n}\left(t^{l^{\prime}} A, t^{l^{\prime}} J\right)\right], E_{n}\left(t^{l^{\prime}} A, t^{l^{\prime}} K\right)\right] \\
& \left.=\left[{ }^{y^{-1} x} E_{n}\left(t^{l^{\prime}} A, t^{l^{\prime}} I\right),{ }^{y^{-1} x} E_{n}\left(t^{l^{\prime}} A, t^{l^{\prime}} J\right)\right],{ }^{y^{-1} x} E_{n}\left(t^{l^{\prime}} A, t^{l^{\prime}} K\right)\right],
\end{aligned}
$$


where by definition $y^{-1} x \in E_{n}\left(\frac{A}{t^{0}}, \frac{A}{t^{0}}\right)$. By (2) in Lemma 7 , for any given integer $l$, we may find a sufficiently large $l^{\prime}$, such that

$$
y^{-1} x\left[\left[E_{n}\left(t^{l^{\prime}} A, t^{l^{\prime}} I\right), E_{n}\left(t^{l^{\prime}} A, t^{l^{\prime}} J\right)\right], E_{n}\left(t^{l^{\prime}} A, t^{l^{\prime}} K\right)\right] \subseteq\left[\left[E_{n}\left(t^{l} A, t^{l} I\right), E_{n}\left(t^{l} A, t^{l} J\right)\right], E_{n}\left(t^{l} A, t^{l} K\right)\right] .
$$

This shows that for any given $l$, one may find a sufficiently large $p_{1}$ such that the first factor of (8)

$$
y^{-1} x\left[\left[e_{1}^{-1},{ }^{c} e_{i^{\prime}, k}\left(-t^{p^{\prime}}\right)\right],{ }^{c} e_{k, j^{\prime}}\left(\frac{\gamma}{t^{m+p^{\prime}}}\right)\right] \in\left[\left[E_{n}\left(t^{l} A, t^{l} I\right), E_{n}\left(t^{l} A, t^{l} J\right)\right], E_{n}\left(t^{l} A, t^{l} K\right)\right] .
$$

Next we consider the second factor of (8),

$$
y^{-1} z\left[\left[{ }^{c} e_{k, j^{\prime}}\left(\frac{-\gamma}{t^{m+p^{\prime}}}\right), e_{1}\right],{ }^{c} e_{i^{\prime}, k}\left(-t^{p^{\prime}}\right)\right] .
$$

Set $p_{2}=p$. Note that

$$
e_{1} \in\left[E_{n}\left(t^{p_{2}} I\right), E_{n}(A, J)\right] \subseteq \mathrm{GL}_{n}\left(A, t^{p_{2}}(I J+J I)\right)
$$

and

$$
{ }^{c} e_{k, j^{\prime}}\left(\frac{\gamma}{t^{m+p^{\prime}}}\right) \in{ }^{E_{n}^{1}\left(\frac{A}{t^{m+p^{\prime}}}\right)} E_{n}^{1}\left(\frac{K}{t^{m+p^{\prime}}}\right),
$$

where $p^{\prime}$ is given by (9) from the first part of the proof. We may apply Lemma 12 to find a sufficiently large $p_{2}$ such that

$$
\left[{ }^{c} e_{k, j^{\prime}}\left(\frac{-\gamma}{t^{m+p^{\prime}}}\right), e_{1}\right] \in \mathrm{GL}_{n}\left(A, t^{p^{\prime \prime}}(K(I J+J I)+(I J+J I) K)\right)
$$

for any given $p^{\prime \prime}$. Using the commutator formula together with (9), one gets

$$
y^{-1} z\left[\left[{ }^{c} e_{k, j^{\prime}}\left(\frac{-\gamma}{t^{m+p^{\prime}}}\right), e_{1}\right],{ }^{c} e_{i^{\prime}, k}\left(-t^{p^{\prime}}\right)\right] \in{ }^{y^{-1} z} E_{n}\left(A, t^{p^{\prime \prime}}(K(I J+J I)+(I J+J I) K)\right)
$$

Applying Lemma 10 twice, one gets

$$
\begin{aligned}
E_{n}\left(A, t^{p^{\prime \prime}}(K(I J+J I)+(I J+J I) K)\right) & \subseteq\left[E_{n}\left(t^{\left\lfloor\frac{2 p^{\prime \prime}}{3}\right\rfloor}((I J+J I)+(I J+J I))\right), E_{n}\left(t^{\left\lfloor\frac{p^{\prime \prime}}{3}\right\rfloor} K\right)\right] \\
& \subseteq\left[\left[E_{n}\left(t^{\left\lfloor\frac{p^{\prime \prime}}{3}\right\rfloor} I\right), E_{n}\left(t^{\left\lfloor\frac{p^{\prime \prime}}{3}\right\rfloor} J\right)\right], E_{n}\left(t^{\left\lfloor\frac{p^{\prime \prime}}{3}\right\rfloor} K\right)\right] .
\end{aligned}
$$

Hence, we have

$$
\begin{aligned}
y^{-1} z\left[\left[{ }^{c} e_{k, j^{\prime}}\left(\frac{-\gamma}{t^{m+p^{\prime}}}\right), e_{1}\right],{ }^{c} e_{i^{\prime}, k}\left(-t^{p^{\prime}}\right)\right] & \subseteq y^{-1} z\left[\left[E_{n}\left(t^{\left\lfloor\frac{p^{\prime \prime}}{3}\right\rfloor} I\right), E_{n}\left(t^{\left.t^{p^{\prime \prime}}\right\rfloor} J\right)\right], E_{n}\left(t^{\left\lfloor\frac{p^{\prime \prime}}{3}\right\rfloor} K\right)\right] \\
& =\left[\left[y^{-1} z E_{n}\left(t^{\left\lfloor\frac{p^{\prime \prime}}{3}\right\rfloor} I\right),{ }^{-1} z E_{n}\left(t^{\left\lfloor\frac{p^{\prime \prime}}{3}\right\rfloor} J\right)\right],{ }^{y^{-1} z} E_{n}\left(t^{\left\lfloor\frac{p^{\prime \prime}}{3}\right\rfloor} K\right)\right] .
\end{aligned}
$$

Now applying (2) in Lemma 7 to every components of the commutator above, we may find a sufficiently large $p^{\prime \prime}$ such that for any given $l$,

$$
\left[\left[y^{-1} z E_{n}\left(t^{\left\lfloor\frac{p^{\prime \prime}}{3}\right\rfloor} I\right),{ }^{y^{-1} z} E_{n}\left(t^{\left\lfloor\frac{p^{\prime \prime}}{3}\right\rfloor} J\right)\right],,^{-1} z E_{n}\left(t^{\left\lfloor\frac{p^{\prime \prime}}{3}\right\rfloor} K\right)\right] \subseteq\left[\left[E_{n}\left(t^{l} A, t^{l} I\right), E_{n}\left(t^{l} A, t^{l} J\right)\right], E_{n}\left(t^{l} A, t^{l} K\right)\right] .
$$

Choose $p_{2}$ in (10) according to this $p^{\prime \prime}$ and then consider $p$ to be the largest among $p_{1}$ and $p_{2}$. This finishes the Lemma. 


\section{MAIN RESUlt}

Now we are in a position to prove the main result of the paper, namely the multiple commutator formulas. We first study the 3 -folded commutator formula. The $n$-folded commutator formula is an easy application of the following theorem. Note that so far most of the conjugation calculus has been performed in $A_{t}$. Using the fact that for a suitable positive integer $l$, by Lemma 5 , the restriction of $\theta_{t}$ to $\mathrm{GL}_{n}\left(A, t^{l} A\right)$ induces an injective homomorphism $\theta_{t}: \mathrm{GL}_{n}\left(A, t^{l} A\right) \rightarrow \mathrm{GL}_{n}\left(A_{t}\right)$, we are able to "pull back" the elements into the group $\mathrm{GL}_{n}(A)$. This will be used in the Theorem 14.

Theorem 14. Let $A$ be a quasi-finite R-algebra and $I, J, K$ be two-sided ideals of $A$. Then for $n \geq 3$,

$$
\left[\left[E_{n}(A, I), \mathrm{GL}_{n}(A, J)\right], \mathrm{GL}_{n}(A, K)\right]=\left[\left[E_{n}(A, I), E_{n}(A, J)\right], E_{n}(A, K)\right]
$$

Proof. The functors $E_{n}$ and $\mathrm{GL}_{n}$ commute with direct limits. By Proposition 4 and $\S 1.5$, one reduces the proof to the case $A$ is finite over $R$ and $R$ is Noetherian.

First by the generalized commutator formula, we have

$$
\left[E_{n}(A, I), \mathrm{GL}_{n}(A, J)\right]=\left[E_{n}(A, I), E_{n}(A, J)\right] .
$$

Thus it suffices to prove the following equation

$$
\left[\left[E_{n}(A, I), E_{n}(A, J)\right], \mathrm{GL}_{n}(A, K)\right]=\left[\left[E_{n}(A, I), E_{n}(A, J)\right], E_{n}(A, K)\right] .
$$

By Lemma 11, $\left[E_{n}(A, I), E_{n}(A, J)\right]$ is generated by the conjugates of the following four types of elements

$$
e=\left[e_{j, i}(\alpha),{ }^{e_{i, j}(r)} e_{j, i}(\beta)\right], \quad e=\left[e_{j, i}(\alpha), e_{i, j}(\beta)\right], \quad e=e_{i, j}(\alpha \beta), \quad \text { and } e=e_{i, j}(\beta \alpha),
$$

where $i \neq j, \alpha \in I, \beta \in J$. We claim that for any $g \in E_{n}(A, K)$,

$$
[e, g] \in\left[\left[E_{n}(A, I), E_{n}(A, J)\right], E_{n}(A, K)\right] .
$$

For any maximal ideal $\mathfrak{m}_{l} \triangleleft R$, choose a $t_{l} \in R \backslash \mathfrak{m}_{l}$ and an arbitrary positive integer $p_{l}$. (We will later choose $p_{k}$ according to Lemma 13.) Since the collection of all $t_{l}^{p_{l}}$ is not contained in any maximal ideal, we may find a finite number of $t_{l}$ and $x_{l} \in R, l=1, \ldots, k$ (relabeling if necessary) such that

$$
\sum_{l} t_{l}^{p_{l}} x_{l}=1
$$

First we take the generators of the first kind, namely the conjugates of $e=\left[e_{j, i}(\alpha),{ }^{e_{i, j}(r)} e_{j, i}(\beta)\right]$. Consider

$$
e=\left[e_{j, i}(\alpha),{ }^{e_{i, j}(r)} e_{j, i}(\beta)\right]=\left[e_{j, i}\left(\left(\sum_{l} t_{l}^{p_{l}} x_{l}\right) \alpha\right),{ }^{e_{i, j}(r)} e_{j, i}(\beta)\right]=\left[\prod_{l} e_{j, i}\left(t_{l}^{p_{l}} x_{l} \alpha\right),{ }^{e_{i, j}(r)} e_{j, i}(\beta)\right] .
$$

By $\left(\mathrm{C} 2^{+}\right)$identity, $e=\left[\prod_{l} e_{j, i}\left(t_{l}^{p_{l}} x_{l} \alpha\right),{ }^{e_{i, j}(r)} e_{j, i}(\beta)\right]$ can be written as a product of the following form:

$$
e=\left(e_{1}\left[e_{j, i}\left(t_{1}^{p_{1}} x_{1} \alpha\right),{ }^{e_{i, j}(r)} e_{j, i}(\beta)\right]\right)\left(e_{2}\left[e_{j, i}\left(t_{2}^{p_{2}} x_{2} \alpha\right),{ }^{e_{i, j}(r)} e_{j, i}(\beta)\right]\right) \cdots\left(e_{k}\left[e_{j, i}\left(t_{k}^{p_{k}} x_{k} \alpha\right),{ }^{e_{i, j}(r)} e_{j, i}(\beta)\right]\right),
$$

where $e_{1}, e_{2}, \ldots e_{m} \in E_{n}(A)$. Note that all $e_{i}$ 's are products of elementary matrices of the form $e_{j, i}(A)$. Thus $e_{l}=e_{j, i}\left(a_{l}\right), l=1, \ldots, k$, which clearly commutes with $e_{j, i}(a)$ for any $a \in A$. So the commutator (13) equals to

$$
e=\left(\left[e_{j, i}\left(t_{1}^{p_{1}} x_{1} \alpha\right),{ }^{e_{1} e_{i, j}(r)} e_{j, i}(\beta)\right]\right)\left(\left[e_{j, i}\left(t_{2}^{p_{2}} x_{2} \alpha\right),{ }^{e_{2} e_{i, j}(r)} e_{j, i}(\beta)\right]\right) \cdots\left(\left[e_{j, i}\left(t_{k}^{p_{k}} x_{k} \alpha\right),{ }^{e_{k} e_{i, j}(r)} e_{j, i}(\beta)\right]\right) .
$$

Thus

$$
[e, g]=\left[\left[e_{j, i}(\alpha),{ }^{e_{i, j}(r)} e_{j, i}(\beta)\right], g\right]=\left[\left[\prod_{l} e_{j, i}\left(t_{l}^{p_{l}} x_{l} \alpha\right),{ }^{e_{i, j}(r)} e_{j, i}(\beta)\right], g\right]
$$


Using $\left(\mathrm{C}^{+}\right)$and in view of (14) we obtain that $[e, g]$ is a product of the conjugates in $E_{n}(A)$ of

$$
\left[\left[e_{j, i}\left(t_{i^{\prime}}^{p_{i^{\prime}}} x_{i^{\prime}} \alpha\right),{ }^{e_{j, i}\left(a_{l}\right) e_{i, j}(r)} e_{j, i}(\beta)\right], g\right] \text {, }
$$

where $a_{l} \in A$ and $l=1, \ldots, k$.

For any maximal ideal $\mathfrak{m}$ of $R$, the ring $A_{\mathfrak{m}}$ contains $K_{\mathfrak{m}}$ as an ideal. Consider the natural homomorphism $\theta_{\mathfrak{m}}: A \rightarrow A_{\mathfrak{m}}$ which induces a homomorphism (call it again $\theta_{\mathfrak{m}}$ ) on the level of general linear groups, $\theta_{\mathfrak{m}}: \mathrm{GL}_{n}(A) \rightarrow \mathrm{GL}_{n}\left(A_{\mathfrak{m}}\right)$. Therefore, for $g \in \mathrm{GL}_{n}(A, K), \theta_{\mathfrak{m}}(g) \in \mathrm{GL}_{n}\left(A_{\mathfrak{m}}, K_{\mathfrak{m}}\right)$. Since $A_{\mathfrak{m}}$ is module finite over the local ring $R_{\mathfrak{m}}, A_{\mathfrak{m}}$ is semilocal [4, $\operatorname{III}(2.5),(2.11)$ ], therefore its stable rank is 1 . It follows that $\mathrm{GL}_{n}\left(A_{\mathfrak{m}}, K_{\mathfrak{m}}\right)=E_{n}\left(A_{\mathfrak{m}}, K_{\mathfrak{m}}\right) \mathrm{GL}_{1}\left(A_{\mathfrak{m}}, K_{\mathfrak{m}}\right)$ (see [11, Th. 4.2.5]). So $\theta_{\mathfrak{m}}(g)$ can be decomposed as $\theta_{\mathfrak{m}}(g)=\varepsilon h$, where $\varepsilon \in E_{n}\left(A_{m}, K_{m}\right)$ and $h$ is a diagonal matrix all of whose diagonal coefficients are 1 , except possibly the $k$-th diagonal coefficient, and $k$ can be chosen arbitrarily.

By (§1.5), we may reduce the problem to the case $A_{t}$ with $t \in R \backslash \mathfrak{m}$. Namely $\theta_{t}(g)$ is a product of $\varepsilon$ and $h$, where $\varepsilon \in E_{n}\left(A_{t}, K_{t}\right)$, and $h$ is a diagonal matrix with only one non-trivial diagonal entry which lies in $A_{t}$. Note that all $\left[e_{j, i}\left(t_{i^{\prime}}^{p^{i^{\prime}}} x_{i^{\prime}} \alpha\right),{ }^{e_{j, i}\left(a_{l}\right) e_{i, j}(r)} e_{j, i}(\beta)\right]$ with $i^{\prime}=1, \ldots, k$ differ from the identity matrix at only the $i, j$ rows and the $i, j$ columns. By the assumption $n>2$, we may choose $h$ so that it commutes with $\left[e_{j, i}\left(t_{i^{\prime}}^{p_{i^{\prime}}} x_{i^{\prime}} \alpha\right),{ }^{e_{j, i}\left(a_{l}\right) e_{i, j}(r)} e_{j, i}(\beta)\right]$. This allows us to reduce our consideration to the case

$$
\left[\left[e_{j, i}\left(t_{i^{\prime}}^{p_{i^{\prime}}} x_{k} \alpha\right),{ }^{e_{j, i}\left(a_{l}\right) e_{i, j}(r)} e_{j, i}(\beta)\right], \varepsilon\right] .
$$

By Lemma 13, one gets that for any given $l_{i^{\prime}}$, there is a sufficiently large $p_{i^{\prime}}$ for every $i^{\prime}=1, \ldots k$, such that

$$
\left[\left[e_{j, i}\left(t_{i^{\prime}}^{p_{i^{\prime}}} x_{k} \alpha\right), e_{j, i}^{e_{j}\left(a_{l}\right) e_{i, j}(r)} e_{j, i}(\beta)\right], \varepsilon\right] \in\left[\left[E_{n}\left(A, t^{l_{i^{\prime}}} I\right), E_{n}\left(A, t^{l_{i^{\prime}} J}\right)\right], E_{n}\left(A, t^{l_{i^{\prime}}} K\right)\right] .
$$

Let's choose every $l_{i}$ to be large enough so that the restriction of $\theta_{t}: G L_{n}\left(t^{l_{i^{\prime}}} A\right) \rightarrow G L_{n}\left(A_{t}\right)$ is injective. Then for every $i^{\prime}$, we have

$$
\left[\left[e_{j, i}\left(t_{i^{\prime}}^{p_{i^{\prime}}} x_{k} \alpha\right),{ }^{e_{j, i}\left(a_{l}\right) e_{i, j}(r)} e_{j, i}(\beta)\right], \varepsilon\right] \in\left[\left[E_{n}(A, I), E_{n}(A, J)\right], E_{n}(A, K)\right] .
$$

Hence $[e, g] \in\left[\left[E_{n}(A, I), E_{n}(A, J)\right], E_{n}(A, K)\right]$.

When the generator is of the second kind, $e=\left[e_{i, j}(\alpha), e_{j, i}(\beta)\right]$, a similar argument goes through, which is left to the reader.

Now consider the generators of the 3rd and 4th kind, namely, the conjugates of the following two types of elements,

$$
e=e_{i, j}(\alpha \beta), \quad \text { or } e=e_{i, j}(\beta \alpha) .
$$

By the normality of $E_{n}(A, I J+J I)$, the conjugates of $e$ are in $E_{n}(A, I J+J I)$. Then

$$
[e, g] \in\left[E_{n}(A, I J+J I), \mathrm{GL}_{n}(A, K)\right] .
$$

By the generalized commutator formula, one obtains

$$
\left[E_{n}(A, I J+J I), \mathrm{GL}_{n}(A, K)\right]=\left[E_{n}(A, I J+J I), E_{n}(A, K)\right] .
$$

Now applying Lemma 10, we finally get

$$
\left[E_{n}(A, I J+J I), E_{n}(A, K)\right] \subseteq\left[\left[E_{n}(A, I), E_{n}(A, J)\right], E_{n}(A, K)\right] .
$$

Therefore $[e, g] \in\left[\left[E_{n}(A, I), E_{n}(A, J)\right], E_{n}(A, K)\right]$. This proves our claim.

Let $e \in\left[E_{n}(A, I), \mathrm{GL}_{n}(A, J)\right]$, and $g \in \mathrm{GL}_{n}(A, K)$. Then by Lemma 11,

$$
e={ }^{c_{1}} e_{1} \times{ }^{c_{2}} e_{2} \times \cdots \times{ }^{c_{k}} e_{k}
$$


with $c_{i} \in E_{n}(A)$ and $e_{i}$ takes the form in (6). Thanks to $\left(\mathrm{C}^{+}\right)$identity, it suffices to show that

$$
\left[{ }^{c_{1}} e_{1}, g\right] \in\left[\left[E_{n}(A, I), E_{n}(A, J)\right], E_{n}(A, K)\right] .
$$

The normality of $E_{n}$ and $\mathrm{GL}_{n}$ groups reduces the problem to show that

$$
\left[e_{i}, g\right] \in\left[\left[E_{n}(A, I), E_{n}(A, J)\right], E_{n}(A, K)\right], \quad i=1, \ldots, k .
$$

But this exactly what has been shown above. This completes the proof.

Corollary 15. Let $A$ be a quasi-finite ring with identity and $I_{i}, i=0, \ldots, m$, be two-sided ideals of $A$. Then

$$
\begin{aligned}
{\left[E_{n}\left(A, I_{0}\right), \mathrm{GL}_{n}\left(A, I_{1}\right), \mathrm{GL}_{n}\left(A, I_{2}\right), \ldots, \mathrm{GL}_{n}\left(A, I_{m}\right)\right] } \\
=\left[E_{n}\left(A, I_{0}\right), E_{n}\left(A, I_{1}\right), E_{n}\left(A, I_{2}\right), \ldots, E_{n}\left(A, I_{m}\right)\right] .
\end{aligned}
$$

Proof. We prove the statement by induction. For $i=1$ this is the generalized commutator formula

$$
\left[E_{n}\left(A, I_{0}\right), \mathrm{GL}_{n}\left(A, I_{1}\right)\right]=\left[E_{n}\left(A, I_{0}\right), E_{n}\left(A, I_{1}\right)\right]
$$

which was proved in [13]. For $i=2$, this is proved in Theorem 14 which will be the first step of induction. Suppose the statement is valid for $i=m-1$ (i.e., there are $m$ ideals in the commutator formula). To prove (15), using Theorem 14, we have

$$
\begin{aligned}
{\left[\left[\left[E_{n}\left(A, I_{0}\right), \mathrm{GL}_{n}\left(A, I_{1}\right)\right], \mathrm{GL}_{n}\left(A, I_{2}\right)\right], \mathrm{GL}_{n}\left(A, I_{3}\right), \ldots, \mathrm{GL}_{n}\left(A, I_{m}\right)\right]=} \\
{\left[\left[\left[E_{n}\left(A, I_{0}\right), E_{n}\left(A, I_{1}\right)\right], E_{n}\left(A, I_{2}\right)\right], \mathrm{GL}_{n}\left(A, I_{3}\right), \ldots, \mathrm{GL}_{n}\left(A, I_{m}\right)\right] . }
\end{aligned}
$$

By Lemma 10, $\left[E_{n}\left(A, I_{0}\right), E_{n}\left(A, I_{1}\right)\right] \subseteq \mathrm{GL}_{n}\left(A, I_{0} I_{1}+I_{1} I_{0}\right)$. Thus

$$
\begin{aligned}
& {\left[\left[\left[E_{n}\left(A, I_{0}\right), E_{n}\left(A, I_{1}\right)\right], E_{n}\left(A, I_{2}\right)\right], \mathrm{GL}_{n}\left(A, I_{3}\right), \ldots, \mathrm{GL}_{n}\left(A, I_{m}\right)\right] \subseteq } \\
& {\left[\left[\mathrm{GL}_{n}\left(A, I_{0} I_{1}+I_{1} I_{0}\right), E_{n}\left(A, I_{2}\right)\right], \mathrm{GL}_{n}\left(A, I_{3}\right), \ldots, \mathrm{GL}_{n}\left(A, I_{m}\right)\right] . }
\end{aligned}
$$

Since there are $m$ groups involved in the commutator subgroups in the right hand side, by induction we get

$$
\begin{aligned}
{\left[\left[\mathrm{GL}_{n}\left(A, I_{0} I_{1}+I_{1} I_{0}\right), E_{n}\left(A, I_{2}\right)\right]\right.} & \left., \operatorname{GL}_{n}\left(A, I_{3}\right), \ldots, \mathrm{GL}_{n}\left(A, I_{m}\right)\right]= \\
& {\left[\left[E_{n}\left(A, I_{0} I_{1}+I_{1} I_{0}\right), E_{n}\left(A, I_{2}\right)\right], E_{n}\left(A, I_{3}\right), \ldots, E_{n}\left(A, I_{m}\right)\right] . }
\end{aligned}
$$

Finally again by Lemma $10, E_{n}\left(A, I_{0} I_{1}+I_{1} I_{0}\right) \subseteq\left[E_{n}\left(A, I_{0}\right), E_{n}\left(A, I_{1}\right)\right]$. Replacing this in the above equation we obtain that the left hand side of (15) is contained in the right hand side. The opposite inclusion is obvious. This completed the proof.

The following corollary shows in fact it doesn't matter where the elementary subgroup appears in the multiple commutator formula.

Corollary 16. Let $A$ be a quasi-finite ring with identity and $I_{i}, i=0, \ldots, m$, be two-sided ideals of $A$. Let $G_{i}$ be subgroups of $\mathrm{GL}_{n}(A)$ such that

$$
E_{n}\left(A, I_{i}\right) \subseteq G_{i} \subseteq \mathrm{GL}_{n}\left(A, I_{i}\right), \quad \text { for } i=0, \ldots, m .
$$

If there is an index $j$ such that $G_{j}=E_{n}\left(A, I_{j}\right)$, then

$$
\left[G_{0}, G_{1}, \ldots, G_{m}\right]=\left[E_{n}\left(A, I_{0}\right), E_{n}\left(A, I_{1}\right), E_{n}\left(A, I_{2}\right), \ldots, E_{n}\left(A, I_{m}\right)\right] .
$$


Proof. Define two-sided ideals of $A$ inductively as follows

$$
\begin{aligned}
& \mathcal{J}_{0}=I_{0} \\
& \mathcal{J}_{k}=I_{k} \mathcal{J}_{k-1}+\mathcal{J}_{k-1} I_{k},
\end{aligned}
$$

where $k=1, \ldots, m$. Now the proof of the lemma divides into several cases:

If $j=0$, the proof follows directly from Corollary 15 . If $j=1$, by a basic property of 2 -fold commutator subgroups, $\left[G_{0}, E_{n}\left(A, I_{1}\right)\right]=\left[E_{n}\left(A, I_{1}\right), G_{0}\right]$, so we reduce the problem to the case of $j=0$.

When $j=k$ with $k \geq 2$, we have

$$
\begin{aligned}
{\left[G_{0}, G_{1}, \ldots, G_{k}, G_{k+1}, \ldots, G_{m}\right] } & =\left[\left[G_{0}, G_{1}, \ldots G_{k}\right], G_{k+1}, \ldots, G_{m}\right] \\
& =\left[\left[G_{0}, G_{1}, \ldots, G_{k-1}, E_{n}\left(A, I_{k}\right)\right], G_{k+1}, \ldots, G_{m}\right]
\end{aligned}
$$

Furthermore, $\left[G_{0}, G_{1}, \ldots, E_{n}\left(A, I_{k}\right)\right]=\left[\left[G_{0}, G_{1}, \ldots, G_{k-1}\right], E_{n}\left(A, I_{k}\right)\right]$, and it follows from Lemma 12, by putting $t=1$, that $\left[G_{0}, G_{1}, \ldots, G_{k-1}\right] \subseteq \operatorname{GL}_{n}\left(A, \mathcal{J}_{k-1}\right)$. By the generalized commutator formula and Lemma 10 we have

$$
\begin{aligned}
{\left[\left[E_{n}\left(A, I_{0}\right), E_{n}\left(A, I_{1}\right), \ldots, E_{n}\left(A, I_{k-1}\right)\right], E_{n}\left(A, I_{k}\right)\right] } & \subseteq\left[\left[G_{0}, G_{1}, \ldots G_{k-1}\right], E_{n}\left(A, I_{k}\right)\right] \\
& \subseteq\left[\mathrm{GL}_{n}\left(A, \mathcal{J}_{k-1}\right), E_{n}\left(A, I_{k}\right)\right] \\
& =\left[E_{n}\left(A, \mathcal{J}_{k-1}\right), E_{n}\left(A, I_{k}\right)\right] \\
& \subseteq\left[\left[E_{n}\left(A, I_{0}\right), E_{n}\left(A, I_{1}\right), \ldots, E_{n}\left(A, I_{k-1}\right)\right], E_{n}\left(A, I_{k}\right)\right] .
\end{aligned}
$$

So

$$
\left[G_{0}, G_{1}, \ldots, G_{k-1}, E_{n}\left(A, I_{k}\right)\right]=\left[\left[E_{n}\left(A, I_{0}\right), E_{n}\left(A, I_{1}\right), \ldots, E_{n}\left(A, I_{k-1}\right)\right], E_{n}\left(A, I_{k}\right)\right],
$$

and therefore

$$
\left[G_{0}, G_{1}, \ldots, G_{m}\right]=\left[E_{n}\left(A, I_{0}\right), E_{n}\left(A, I_{1}\right), \ldots, E_{n}\left(A, I_{k-1}\right), E_{n}\left(A, I_{k}\right), G_{k+1}, \ldots, G_{m}\right] .
$$

Finally, we finish the proof by applying Corollary 15.

We finish the paper by a most general multiple commutator formula. Note that taking a commutator is a binary operation, and for $G_{1}, \ldots, G_{n}, n \geq 3$, there any many ways to insert the commutator brackets [_, - ] to make the sequence into a meaningful multi-commutator expression. For example for $n=4$, we can have the following two arrangements $\left[\left[G_{0},\left[G_{1}, G_{2}\right]\right], G_{3}\right]$ and $\left[\left[G_{0}, G_{1}\right],\left[G_{2}, G_{3}\right]\right]$ among many others. We denote by $\llbracket G_{0}, G_{1}, \ldots, G_{m} \rrbracket$ a "meaningful" multi-commutator formula.

Theorem 17. Let $A$ be a quasi-finite ring with identity and $I_{i}, i=0, \ldots, m$, be two-sided ideals of $A$. Let $G_{i}$ be subgroups of $\mathrm{GL}_{n}(A)$ such that

$$
E_{n}\left(A, I_{i}\right) \subseteq G_{i} \subseteq \mathrm{GL}_{n}\left(A, I_{i}\right), \quad \text { for } i=0, \ldots, m .
$$

If there is an index $j$ such that $G_{j}=E_{n}\left(A, I_{j}\right)$, then

$$
\llbracket G_{0}, G_{1}, \ldots, G_{m} \rrbracket=\llbracket E_{n}\left(A, I_{0}\right), E_{n}\left(A, I_{1}\right), \ldots, E_{n}\left(A, I_{m}\right) \rrbracket .
$$

Proof. For simplicity denote $E_{n}\left(A, I_{i}\right)$ by $E_{i}$. The proof is by induction on $m$. For $m=0$ and $m=1$ there is nothing to prove. For $\mathrm{m}=2$, the commutator $\llbracket G_{0}, G_{1}, G_{2} \rrbracket$ can take one of the the forms

(1) $\left[\left[G_{0}, G_{1}\right], E_{2}\right]$,
(2) $\left[E_{0},\left[G_{1}, G_{2}\right]\right]$,
(3) $\left[\left[E_{0}, G_{1}\right], G_{2}\right]$,
(4) $\left[\left[G_{0}, E_{1}\right], G_{2}\right]$, 
(5) $\left[G_{0},\left[E_{1}, G_{2}\right]\right]$,

(6) $\left[G_{0},\left[G_{1}, E_{2}\right]\right]$.

Since by $(\mathrm{C}(6))$, for two subgroups $H$ and $K$, we have $[H, K]=[K, H]$, we can reduce the cases $(1)$ and (2) and (3)-(6) to each other, respectively, and therefore it is enough to prove the theorem for the cases (1) and (3). For the first arrangement (1), using Lemma 12, for $t=1$, Lemma 10 and the generalized commutator formula we have

$$
\begin{aligned}
\llbracket E_{0}, E_{1}, E_{2} \rrbracket=\left[\left[E_{0}, E_{1}\right], E_{2}\right] & \subseteq\left[\left[G_{0}, G_{1}\right], E_{2}\right] \\
& \subseteq\left[\left[\operatorname{GL}_{n}\left(A, I_{0}\right), \operatorname{GL}_{n}\left(A, I_{1}\right)\right], E_{n}\left(A, I_{2}\right)\right] \\
& \subseteq\left[\operatorname{GL}_{n}\left(A, I_{0} I_{1}+I_{1} I_{0}\right), E_{n}\left(A, I_{2}\right)\right] \\
& =\left[E_{n}\left(A, I_{0} I_{1}+I_{1} I_{0}\right), E_{n}\left(A, I_{2}\right)\right] \\
& \subseteq\left[\left[E_{n}\left(A, I_{0}\right), E_{n}\left(A, I_{1}\right)\right], E_{n}\left(A, I_{2}\right)\right] \\
& =\left[\left[E_{0}, E_{1}\right], E_{2}\right]=\llbracket E_{0}, E_{1}, E_{2} \rrbracket .
\end{aligned}
$$

This shows that $\llbracket G_{0}, G_{1}, G_{2} \rrbracket=\llbracket E_{0}, E_{1}, E_{2} \rrbracket$. The arrangement (3) (and therefore (4)-(6)) follows immediately from Theorem 14 .

For the main step of induction, we consider two cases. Suppose first there is a mixed commutator $\left[G_{i}, G_{i+1}\right]$ in $\llbracket G_{0}, G_{1}, \ldots, G_{m} \rrbracket$, where neither $G_{i}$ nor $G_{i+1}$ is the fixed elementary subgroup $E_{j}$. Then

$$
\begin{aligned}
\llbracket G_{0}, G_{1}, \ldots, G_{m} \rrbracket & =\llbracket G_{0}, G_{1}, \ldots,\left[G_{i}, G_{i+1}\right], \ldots, G_{m} \rrbracket \\
& \subseteq \llbracket G_{0}, G_{1}, \ldots,\left[\operatorname{GL}_{n}\left(A, I_{i}\right), \operatorname{GL}_{n}\left(A, I_{i+1}\right)\right], \ldots, G_{m} \rrbracket \\
& \subseteq \llbracket G_{0}, G_{1}, \ldots, \operatorname{GL}_{n}\left(A, I_{i} I_{i+1}+I_{i+1} I_{i}\right), \ldots, G_{m} \rrbracket .
\end{aligned}
$$

Note that there are one fewer ideal involved in the last commutator formula (i.e., $m-1$ ideals) which also contains an elementary subgroup, and so by induction

$$
\begin{aligned}
\llbracket G_{0}, G_{1}, \ldots, \mathrm{GL}_{n}\left(A, I_{i} I_{i+1}+I_{i+1} I_{i}\right), \ldots, G_{m} \rrbracket & =\llbracket E_{0}, E_{1}, \ldots, E_{n}\left(A, I_{i} I_{i+1}+I_{i+1} I_{i}\right), \ldots, E_{m} \rrbracket \\
& \subseteq \llbracket E_{0}, E_{1}, \ldots,\left[E_{n}\left(A, I_{i}\right), E_{n}\left(A, I_{i+1}\right)\right], \ldots, E_{m} \rrbracket \\
& =\llbracket E_{0}, E_{1}, \ldots, E_{m} \rrbracket .
\end{aligned}
$$

Putting 16 and 17 together, we get

$$
\llbracket G_{0}, G_{1}, \ldots, G_{m} \rrbracket=\llbracket E_{0}, E_{1}, \ldots, E_{m} \rrbracket .
$$

For the remaining case, suppose now that if there is a mixed commutator of the form $\left[G_{i}, G_{i+1}\right]$, in $\llbracket G_{0}, G_{1}, \ldots, G_{m} \rrbracket$, then one of $G_{i}$ or $G_{i+1}$ is our fixed elementary subgroup $E_{j}$. Write

$$
\llbracket G_{0}, G_{1}, \ldots, G_{m} \rrbracket=\left[\llbracket G_{0}, G_{1}, \ldots, G_{k} \rrbracket, \llbracket G_{k+1}, \ldots, G_{m} \rrbracket\right] .
$$

Since the fixed elementary subgroup $E_{j}$ is in one of the factors, one of $\llbracket G_{0}, G_{1}, \ldots, G_{k} \rrbracket$ or $\llbracket G_{k+1}, \ldots, G_{m} \rrbracket$ has to have a mixed commutator of the form $\left[G_{i^{\prime}}, G_{i^{\prime}+1}\right]$ with neither $G_{i^{\prime}}$ nor $G_{i^{\prime}+1}$ the fixed $E_{j}$, which has been excluded from the outset. This forces $k=0$ or $k=m-1$, i.e.,

$$
\begin{aligned}
\llbracket G_{0}, G_{1}, \ldots, G_{m} \rrbracket & =\left[G_{0}, \llbracket G_{1}, \ldots, G_{m} \rrbracket\right], \text { or } \\
\llbracket G_{0}, G_{1}, \ldots, G_{m} \rrbracket & =\left[\llbracket G_{0}, G_{1}, \ldots, G_{m-1} \rrbracket, G_{m}\right] .
\end{aligned}
$$

Repeating this argument, by an easy induction and $(\mathrm{C}(6))$ one can see that because of absence of $\left[G_{i}, G_{i+1}\right]$, the multiple commutator $\llbracket G_{0}, G_{1}, \ldots, G_{m} \rrbracket$ has the standard form (see $\S 1.3$ )

$$
\llbracket G_{0}, G_{1}, \ldots, G_{m} \rrbracket=\left[G_{i_{0}}, G_{i_{1}}, \ldots, G_{i_{m}}\right] .
$$


By Corollary 16

$$
\left[G_{i_{0}}, G_{i_{1}}, \ldots, G_{i_{m}}\right]=\left[E_{i_{0}}, E_{i_{1}}, \ldots, E_{i_{m}}\right] .
$$

Now using $(\mathrm{C}(6))$ again and re-arranging $E_{i}$ in the reverse order we get

This finishes the proof.

$$
\left[E_{i_{0}}, E_{i_{1}}, \ldots, E_{i_{m}}\right]=\llbracket E_{0}, E_{1}, \ldots, E_{m} \rrbracket .
$$

\section{REFERENCES}

[1] A. Bak, Nonabelian $K$-theory: the nilpotent class of $K_{1}$ and general stability, $K$-Theory 4 (1991), 363-397. 2, 4, 5

[2] A. Bak, R. Hazrat, N.A. Vavilov, Localization completion strikes again: relative $K_{1}$ is nilpotent by abelian. J. Pure Appl. Algebra 213 (2009), 1075-1085.

[3] A. Bak, N.A. Vavilov, Normality for elementary subgroup functors, Math. Proc. Camb. Philos. Soc. 118(1) (1995), 35-47.

[4] H. Bass, Algebraic K-theory. Benjamin, New York, 1968. 10

[5] H. Bass, K-theory and stable algebra. Inst. Hautes Etudes Sci., Publ. Math. 22 (1964), 5-60. 1

[6] Z. Borevic, N.A. Vavilov, The distribution of subgroups in the full linear group over a commutative ring, Proc. Steklov Institute Math 3 (1985), 27-46. 1

[7] V.N. Gerasimov, The group of units of the free product of rings. Mat. Sbornik 134 (1987)(1), 42-65.

[8] I.Z. Golubchik, On the general linear group over weakly Noetherian associative rings. Fundam. Appl. Math. 1 (1995)(3), 661-668.

[9] I.Z. Golubchik, A. V. Mikhalev, On the group of elementary matrices over PI-rings. in Investigations in Algebra (Iad. Tbil. Gos. Univ., Tbilisi, 1985), 20-24.

[10] S.G. Khlebutin, Some properties of the elementary subgroup. in Algebra, Logic, and Number Theory (Izd. Mosk. Gos. Univ., Moscow, 1986), 86-90.

[11] A.J. Hahn and O.T. O'Meara. The Classical groups and K-Theory, Springer, 1989. 10

[12] R. Hazrat, N. Vavilov, Bak's work on the K-theory of rings, with an appendix by Max Karoubi, J. K-Theory 4 (2009), 1-65. 2

[13] R. Hazrat, Z. Zhang, Generalized commutator formulas, Comm. in Algebra, 39 (2011), 1441-1454. 2, 4, 11

[14] R. Hazrat, N.A. Vavilov, Z. Zhang, Relative unitary commutator calculus and applications, J. Algebra, to appear, arXiv:0911.5510 2

[15] R. Hazrat, A.V. Stepanov, N.A. Vavilov, Z. Zhang, The yoga of commutators, J. Math. Sci., to appear. 2

[16] A.W. Mason, On subgroup of $\operatorname{GL}_{n}(n, A)$ which are generated by commutators, II. J. reine angew. Math. 322 (1981), 118-135. 1

[17] A.W. Mason, A further note on subgroups of $\operatorname{GL}_{n}(n, A)$ which are generated by commutators. Arch. Math. 37 (1981)(5) 401-405.

[18] A.W. Mason, W.W. Stothers, On subgroup of $\mathrm{GL}_{n}(n, A)$ which are gnerated by commutators. Invent. Math. 23 (1974), 327-346. 1

[19] A.V. Stepanov, On the normal structure of the general linear group over a ring, Zap. Nauch. Sem. POMI 236 (1997), $162-169$.

[20] A.V. Stepanov, N.A. Vavilov, Decomposition of transvections: A theme with variations. K-theory 19 (2000), $109-153$.

[21] A.A. Suslin, On the structure of the special linear group over the ring of polynomials, Izv. Akad. Nauk SSSR, Ser. Mat. $141(1977)(2), 235-253.1$

[22] J. Tits, Systèmes générateurs de groupes de congruence, C. R. Acad. Sci. Paris, Sér A, 283 (1976), 693-695. 5

[23] M.S. Tulenbaev, The Schur multiplier of the group of elementary matrices of finite order. Zap. Nauch. Sem LOMI 86 (1979), 162-169. 1

[24] N.A. Vavilov, A.V. Stepanov, Standard commutator formula. Vestnik St. Petersburg State Univ., ser.1 41 No. 1(2008), 5-8. 1,2

[25] N.A. Vavilov, A.V. Stepanov, Standard commutator formula, revisited. Vestnik St. Petersburg State Univ., ser.1, 43 No. 1 (2010), 12-17. 2

[26] L.N. Vaserstein, On the normal subgroups of $\mathrm{GL}_{n}$ over a ring. Lecture Notes in Math. 854 (1981), 456-465. 1

Department of Pure Mathematics, Queen's University Belfast, Belfast BT7 1NN, Northern Ireland, United Kingdom

E-mail address: r.hazrat@qub.ac.uk

Department of Mathematics, Beijing Institute of Technology, Beijing, China

E-mail address: zuhong@gmail.com 